\title{
Customized Usability Engineering for a Solar Control Unit: Adapting Traditional Methods to Domain and Project Constraints
}

\author{
Patricia Böhm, Tim Schneidermeier, and Christian Wolff \\ University of Regensburg, Department of Media Informatics, Universitätsstrasse 31, \\ 93053 Regensburg, Germany \\ Patricia.Boehmastud.uni-regensburg.de, \\ \{Tim.Schneidermeier, Christian.Wolff\} @sprachlit.uni-regensburg.de
}

\begin{abstract}
This paper describes the adaption and customization of usability engineering methods for the interface design of a solar control unit. The design of a nontraditional interface, constrained access to representative users and a lack of common interface standards were domain-related issues to overcome. Due to limited resources, a Guerilla HCI approach was established. Traditional low-cost methods like prototyping and simplified usability testing were applied and adapted to fit in the domain-specific context. Good feedback indicates suitability of modified discount methods in the new domain.
\end{abstract}

Keywords: usability engineering, discount usability, user-centered design, user interface design, nontraditional user interfaces, facility management.

\section{Introduction}

The advantages of usability testing for the development of well-designed, intuitive and easy to use interactive software have been acknowledged for a long time. Since the rise of smart phones and home entertainment systems, usability engineering found its way into additional application areas [1], [2], [3], [4]. Nevertheless, in many technical domains like facility management usability engineering methods still need to be adapted to fit in the specific context. However domain-specific usability has not been established by research approaches yet [5].

This paper describes a case study of an user-centered design (UCD) approach for designing the user interface of a solar control unit. It focuses on the adaption and customization of established low-cost usability methods to the domain of nontraditional interfaces, i.e. domestic information appliances encompassing its critical constraints and challenges and their influence on the method selection, a more detailed description of the design process and results can be found in [6]. Usability efforts in the field of facility management has to overcome domain-related issues like the design of nontraditional interfaces [7], the lack of common interface standards and limited access to representative users. Constraints of human and financial resources, a common challenge in UCD practice [8], have to be managed. In the context of an interdisciplinary research funded by the Bavarian Ministry of Economic 
Development ${ }^{1}$, the project team consisted of usability engineers of the University of Regensburg and two domain experts of the enterprise emz Hanauer. ${ }^{2}$

In the first section the general approach of customized low-cost usability engineering, it's goals and constraints in the context of a solar control device are pointed out. In section 3 an approach towards prototyping and evaluating a nontraditional interface is shown. This is followed by a description of a card sorting study dealing with the different capabilities of users especially domain knowledge. The last section describes the approach of parallel usability testing. A modified usability testing procedure, including different tasks and simultaneous testing sessions was established in order to compensate limited availability of users.

\section{Customizing Usability Methods}

Usability Engineering can be defined as a set of methodologies and activities used for designing an interactive product [8]. Ideally, the most appropriate methods are selected for best results. Yet reality shows that in most projects many parameters like project resources, access to users, interface properties or return of investment (ROI) have to be respected. Approaches towards discount or guerrilla usability are facing this challenge [9], [10], [11], [12]. The main idea is to use simpler and therefore cheaper methods in constrained contexts. For example Nielsen's discount usability engineering is based on techniques like scenarios and simplified thinking aloud [13]. Due to limited human and financial resources these ideas were also taken account of in the design of the smart sol control unit. The methods were customized and adapted to the domain of nontraditional interfaces. Prototyping and simplified user testing including the thinking aloud method built the basis for our Guerilla HCI approach.

\subsection{Usability Goals and Requirements}

User characteristics and typical tasks were identified by informal interviews with domain experts and potential users of the control unit. Main user groups could be defined as installers and end-users. Differing in terms of usage patterns, expertise in control devices as well as knowledge about solar systems in general, both needs had to be addressed. The craftsman on the one hand is responsible for setting up and attendance of the device, being familiar with this kind of devices in as well as with the according terminology. On the other hand, typical end-users hardly use these devices at all due to hard to use and counter-intuitive interfaces of state of the art control devices. ${ }^{3}$ They are usually not familiar with technical terms or the general functioning of the solar plant or other devices of comparable technical complexity. According to these requirements, two goals had to be accomplished: On the one hand domain specific workflows have to be optimized, and on the other hand, an intuitive and selfexplanatory user interface has to be designed to break down barriers for the end-user.

\footnotetext{
${ }^{1}$ Grant IUK 0910-0003.

${ }^{2} \mathrm{http}: / /$ www.emz-hanauer.de, a medium-sized enterprise specialized in household appliances as well as domestic and environmental engineering, located in Nabburg, Bavaria [accessed February 2011].

${ }^{3}$ Results of market research and user interviews conducted by the authors in 2009/2010.
} 


\subsection{Testing Constraints}

Besides resource limitations, other constraints influenced method selection and adaption. As described above, usability testing had to involve two user groups: end users and installers. Whereas possible end users are more or less easy to reach, e.g. using student volunteers, recruiting installers as test persons is more challenging. Thus an efficient way of parallel usability testing was established to compensate their limited availability.

User interface guidelines and sets of predefined input controls (e.g. mouse, keyboard) limit the design space for desktop interfaces. While each application provides different functionality, consistent controls for standard operations such as opening, closing or setting preferences can be expected. Designing a nontraditional interface involves far more design decisions and challenges including not just the onscreen interface but hardware parts as well. The predictability of consistent controls is completely absent in nontraditional interfaces [14]. Thus we had to modify and extend typical prototyping methods.

\section{Prototyping a Nontraditional Interface}

The primary method chosen for evaluating the design concepts was prototyping. Because of the fast and comparatively easy process, low-fidelity paper prototypes were applied at first [13], [10]. It turned out that they were fine to represent a quick idea of the on-screen layout, but insufficient in all other means regarding nontraditional interfaces: Hardware interaction, physical properties of controls and visual aspects needed to be sketched.

\subsection{Hardware Dummy to Evaluate Overall Design}

Due to time constraints especially regarding the production period the device hardware had to be designed first. Based on a competitor analysis and expert reviews on usability and required human-machine interaction, the hardware design was developed in cooperation with an industrial design company. A full color display was

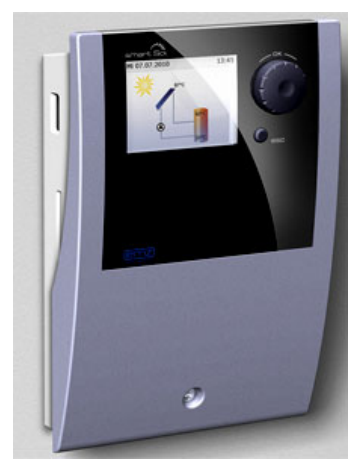

Fig. 1. Overall design concept 
chosen to overcome the technical impression generated by reduced display techniques [15]. The interaction is based on a click wheel for navigation and confirmation and an escape button for going back. Click wheels are appropriate for the fast adjustment of parameters with great values ranges [16]. The escape button guarantees the easy reversal of actions as an important principle for interface design [17]. To get an impression of the physical properties an (almost non-functional) hardware dummy was built. In cooperation with domain experts the basic shape and positioning of the control elements were iteratively evaluated and improved. Thus, design flaws like a lack of height of the click-wheel could be revised in an early stage.

\subsection{Evaluation of Small-Display Layouts with Mockups}

Based on the earlier paper sketches, graphic mockups were used for design and evaluation of the screen layout. In contrast to traditional website or software application wireframes, the layout also had to consider the constraints of smalldisplay characteristics, e.g. visual angle, viewing distance, luminance, character and font attributes, display placement [18]. Thus graphic mock ups were used to communicate our ideas to domain specialists and were reusable for usability testing. Because of the recycling time could be saved. For the evaluation of font, font-size and color schemes, mockups were rendered to imply real size and resolution constraints of the final display.

\subsection{Evaluating Interaction with an HTML-Based Prototype}

The evaluation of the overall interaction design concept was more demanding. Due to time and resource constraints, the concept had to be evaluated without an operational hardware prototype. In addition, interaction had to be modeled without the final information architecture. Final menu structure determination had to be deferred to a later point as representative users were not available at the time.

Vertical interactive html-prototypes proved to be a valid approach to these problems. Two important use cases, the initial setting up of the device and an error handling situation were implemented to address both user groups, installers and end users. In order to simulate a model as realistic as possible despite the given constraints a virtual representation of the device`s body and controls were included. Hence users could interact with the prototype using a virtual click wheel and escape button.

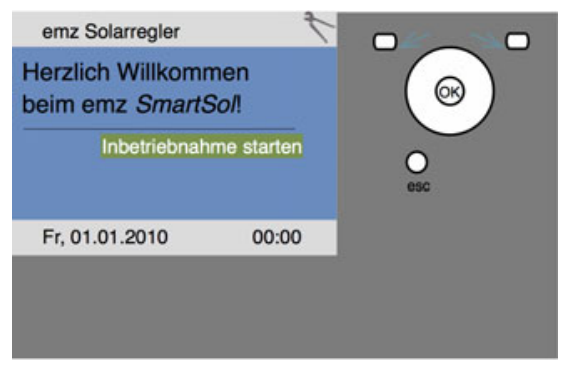

Fig. 2. Interactive html-based prototype 


\section{Card Sorting and Domain Constraints}

41 menu items were extracted out of the functional specification document of the smart sol controller. Organizing them in a logical and intuitive structure that matches both user groups is crucial for the intended self-explanatory and efficient interface. Therefore, a card sorting method was chosen, a distinguished method to create information architecture according to user's metal model [19]. Originally using real paper cards, the method has been translated into an online exercise with virtual cards [20].

\subsection{Failure of Online-Based Open Card Sorting}

Due to the domain-specific terminology and the lack of standards an open card sort seemed to be appropriate: Here, participants have to group items and label the groups themselves [20]. A first attempt using a free online tool ${ }^{4}$ failed. The participants, installers to whom we got in touch through the enterprise as well as randomly chosen students received an e-mail with an invitation and a link to the online tool. The return of installers was very low. Little computer experience and a lack of understanding of the goal might have led to the poor results, probably typical for this kind of design task and test users. In addition, grouping and labeling by end user was very heterogeneous and inconsistent. Therefore, we concluded that finding specific categories for the technical and abstract items is too difficult for non-professionals.

\subsection{Customizing Card Sorting to Domain Constraints}

In a second try we strived to take the specifics of the different target groups into account. To simplify the task, installers and end-users should participate in a closed card sort where categories are predefined. To generate the hyperonyms for the categories on the highest hierarchy level an open card sort with five domain experts of the enterprise using the online tool was conducted. Their labeling was not unambiguous, but the groupings implied a helpful domain-related view. Additionally, we compared their classifications to the menu structures of more or less well-designed competitor devices. For nomenclature of categories and items we tried to strike a balance between popularity, distinctness and domain conventions. Subsequently student volunteers were kindly asked to take part in the closed card sort using the online tool once more. In order to motivate the craftsmen and take their potential lack of computer affinity into account we established a real paper card sort with the presence of test supervisors who could be asked if problems would occur. In order to save time and make the most out of their voluntary participation, the card sorting session of the installers was combined with other usability tests on one evening. The procedure and its constraints are described in the section parallel usability testing below.

\subsection{Simple and Quick Analyzing}

Analyzing the gained data from the conducted card sort was done right after the session. Data from each group was first evaluated separately before the results were

\footnotetext{
${ }^{4}$ WebCAT: http://zing.ncsl.nist.gov/WebTools/WebCAT/overview.html [accessed February 2011].
} 
assembled in a second step. Basically, three different methods for analysis are available: Analysis with a special card sorting software, cluster analysis with a statistical software or frequency detection with spreadsheets [19].

Although the applied online-tool offers rudimentary analysis functions the results of the paper card sorting had also to be included. Therefor we used SPSS for cluster analysis as well as frequency distribution. According to our experience using spreadsheets is a practicable and efficient way. The main idea is calculating for each card and category the percentages of participants that assigned them. Nevertheless frequencies are insufficient when there is no majority and allocation is ambiguous. Therefore cluster analysis can be used to get supplementary clues. From cluster analysis a dendogram can be derived: a tree diagram where the cards that were sorted the most similar by the participants is placed on branches that are close together. Considering the point at which the items join together their similarity can be deduced [20]. Altogether, spreadsheet approach and cluster analysis complement each other fairly well.

\section{Parallel Usability Testing}

All usability tests with installers were conducted on one day, as the craftsmen were not jointly available for a longer period of time. The parallel usability testing including card sorting, user tests as well as a post-test questionnaire required adapted procedures in terms of recruitment of participants, test environment and material.

\subsection{Recruitment of Installers}

Usually test persons for a usability study are recruited by special agencies or through advertisements and get some kind of reward for their participation [21], [22]. Having restricted resources we had to search for others ways of recruitment.

Useful contacts to installers could be established using the enterprise's network and asking them for voluntary participation. To raise willingness for voluntary participation, usability testing was embedded in an informal social event with all participants getting an invitation via mail. The event began with a (rare) guided tour through the production department of the enterprise before usability sessions were started. A get-together with snacks at the end underlined the casual character. Despite these promotional efforts only 11 out of 20 invited installers participated.

\subsection{Test Environment for Simultaneous Testing}

Though testing in the field is the best method to consider factors of context of use, and therefore detect more severe issues [23], [24], it was not practicable in our approach. Using a HTML-prototype as objective, testing in a traditional laboratory environment was the only option.

Due to their voluntary participation usability testing had to be accomplished in a reasonable time. The testing sessions were conducted simultaneously instead of one after another. Therefore two laboratory-like testing environments were established in office accommodations of the enterprise. One room was prepared with laptops for user tests and another room was equipped with tables and paper card sets for card 
sorting. For the simultaneous proceeding additional supervisors were needed. Domain experts of the enterprise and five additional employees, mainly engineers and programmers, were briefed and deployed as additional test supervisors.

\subsection{Test Tasks and Procedure}

In order to make the most out of the voluntary participation of the target group testing sessions included two tasks: Closed card sorting (using real paper cards) with predefined categories and conducting two use cases applying the interactive html prototype. The participants were divided into two groups, each of which accomplished the different tasks in reverse order to minimize statistical impact. To guarantee traceability and to speed up the test process, protocols and documents were prepared in advance for the testing session. Each test person received a portfolio containing instructions, questionnaires and protocol forms already labeled with ids. The card sorting was conducted with two supervisors. After a short introduction the installers were free to start with the sorting. The groupings were left on the table and recorded with a protocol form.

During user tests every participant was supported by a single supervisor and asked to think aloud. All comments were written down in a predefined protocol form. A post-test questionnaire including questions about interaction concept, layout aspects and workflow of test scenarios, completed the test. Altogether parallel usability testing worked out quite well: Engaging domain specialists in usability testing raised efficiency and helped bridging the knowledge gap. Due to their expertise questions on technical details of supported solar systems could be answered. In addition, their domain-related view on actual usability problems was helpful for assessing these issues. No major usability problems were found and the overall interaction design has been approved. The final design of the smart sol control unit was rated as intuitive and well-arranged.

\subsection{Method Limitations}

Due to time pressure user testing was conducted with HTML-based prototypes. Though simulating hardware interaction with virtual elements worked out, this kind of testing objective is limited. Testing the on-screen user interface on the real hardware would for sure have been an enrichment. Nevertheless due to all constraints mentioned above the quick and dirty solution turned out fine in this case. Other important factors like the context of use of the device, usually wall-mounted in a basement-like area, could neither be considered in the test sessions.

\section{Conclusion}

In this paper a customized Guerilla HCI approach used for the development of a solar control with constrained resources is described. Traditional low-cost methods were applied and adapted to fit in the domain-specific context. The smart sol solar control was presented at Intersolar Europe 2010, the world`s largest trade show for solar 
products $^{5}$. The overall design as well as the interaction design in particular were rated a success. This feedback and the positive resonance of user testing indicate that discount usability engineering approaches are suited in domains beyond the desktop. Nevertheless our modifications show that there is a need for domain-specific usability, an area research is just beginning to explore, e. g. in the $1^{\text {st }}$ European Workshop on HCI Design and Evaluation ${ }^{6}$ focusing on the influence of domains Further studies examining the usability of the controller in the field are planned to enlarge our findings.

Acknowledgments. This work has been conducted in the context of the research project Modino that is funded by the Bavarian Ministry of Economic Development (Grant IUK 0910-0003).

\section{References}

1. Hallnäs, L., Redström, J.: From Use to Presence: On Expressing and Aesthetics of Everyday Computational Things. ACM Trans. Comput.-Hum. Interact. 9, 106-124 (2002)

2. Han, S.H., Yun, M.H., Kwahk, J., Hong, S.W.: Usability of Consumer Electronic Products. International Journal of Industrial Ergonomics 28, 143-151 (2001)

3. Petersen, M.G., Madsen, K.H., Kjær, A.: The Usability of Everyday Technology: Emerging and Fading Opportunities. ACM Trans. Comput.-Hum. Interact. 9, 74-105 (2002)

4. Weiss, S.: Handheld Usability. Wiley, New York (2002)

5. Chilana, P.K., Wobbrock, J.O., Ko, A.J.: Understanding usability practices in complex domains. In: Proceedings of the 28th International Conference on Human Factors in Computing Systems, pp. 2337-2346. ACM, Atlanta (2010)

6. Smart Sol - Bringing User Experience to Facility Management: Designing the User Interaction of a Solar Control Unit. To appear in: Proceedings of HCI International 2011 (2011)

7. Kortum, P. (hrsg): HCI Beyond the GUI: Design for Haptic, Speech, Olfactory, and Other Nontraditional Interfaces. Morgan Kaufmann, San Francisco (2008)

8. Vredenburg, K., Mao, J., Smith, P.W., Carey, T.: A survey of user-centered design practice. In: Proceedings of the SIGCHI Conference on Human Factors in Computing Systems: Changing our World, Changing Ourselves, pp. 471-478. ACM, Minneapolis (2002)

9. Kane, D.: Finding a Place for Discount Usability Engineering in Agile Development: Throwing Down the Gauntlet. In: Proceedings of the Conference on Agile Development, p. 40. IEEE Computer Society, Los Alamitos (2003)

10. Snyder, C.: Paper prototyping: the fast and easy way to design and refine user interfaces. Morgan Kaufmann, Amsterdam (2003)

11. Bias, R.G., Mayhew, D.J. (eds.): Cost-Justifying Usability. Academic Press, Inc., Orlando (1994)

12. Nielsen, J., Mack, R.L.: Usability inspection methods. Wiley, New York (1994)

\footnotetext{
5 http://www.intersolar.de [accessed February 2011].

${ }^{6} \mathrm{http}: / /$ sites.google.com/site/ehcide/, taking place in Limassol, Cyprus in April 2011 [accessed February 2011].
} 
13. Nielsen, J.: Guerilla HCI: Using Discount Usability Engineering to Penetrate the Intimidation Barrier. In: Bias, R.G., Mayhew, D.J. (eds.) Cost-Justifying Usability, pp. 245-270. Morgan Kaufmann Publishers, San Francisco (1994)

14. Kortum, P.: Introduction to the Human Factors of Nontraditional Interfaces. In: Kortum, P. (ed.) HCI Beyond the GUI: Design for Haptic, Speech, Olfactory, and Other Nontraditional Interfaces, pp. 1-24. Morgan Kaufmann, San Francisco (2008)

15. Schmidt, A., Terrenghi, L.: Methods and Guidelines for the Design and Development of Domestic Ubiquitous Computing Applications. In: Proceedings of the Fifth IEEE International Conference on Pervasive Computing and Communications, pp. 97-107. IEEE Computer Society, Los Alamitos (2007)

16. Baumann, K., Lanz, H.: Controls. In: Baumann, K., Lanz, H. (eds.) User Interface of Electronic Appliances, pp. 131-161. Taylor \& Francis, New York (2001)

17. Shneiderman, B., Plaisant, C.: Designing the user interface: Strategies for effective humancomputer interaction. Addison-Wesley/Pearson, Upper Saddle River, NJ (2010)

18. Kärkkäinen, L., Laarni, J.: Designing for small display screens. In: Proceedings of the Second Nordic Conference on Human-Computer Interaction, pp. 227-230. ACM, New York (2002)

19. Courage, C., Baxter, K.: Understanding Your Users: A Practical Guide to User Requirements Methods, Tools, and Techniques. Morgan Kaufmann Publishers, San Francisco (2004)

20. Tullis, T.: Measuring the User Experience. Elsevier, Amsterdam (2008)

21. Dumas, J.S., Redish, J.C.: A Practical Guide to Usability Testing. Intellect, Exeter (1999)

22. Rubin, J., Chisnell, D.: Handbook of Usability Testing. How to Plan Design and Conduct Effective Tests. Wiley, Indianapolis (2008)

23. Duh, H.B., Tan, G.C.B., Chen, V.H.: Usability evaluation for mobile device: a comparison of laboratory and field tests. In: Proceedings of the 8th Conference on Human-Computer Interaction with Mobile Devices and Services, pp. 181-186. ACM, Helsinki (2006)

24. Nielsen, C.M., Overgaard, M., Pedersen, M.B., Stage, J., Stenild, S.: It's worth the hassle!: the added value of evaluating the usability of mobile systems in the field. In: Proceedings of the 4th Nordic Conference on Human-Computer Interaction: Changing Roles, pp. 272280. ACM, Oslo (2006) 